\title{
Editorial
}

\section{Burnout und Stress beim Übergang in den Lehrerberuf}

\author{
Friederike Zimmermann, Uta Klusmann
}

Seit über 30 Jahren wird wiederkehrend darauf hingewiesen, dass der Einstieg in den Lehrerberuf eine besonders herausfordernde Phase sei, in der es zu Anpassungsschwierigkeiten der angehenden Lehrkräfte komme (z. B. Tynjälä \& Heikkinen, 2011). Neben Veränderungen in Einstellungen und Überzeugungen sowie dem Erleben von Inkompetenz wird dabei vor allem auf ein erhöhtes Erleben von Stress und Beanspruchung am Berufseinstieg verwiesen (Huberman, 1989; Müller-Fohrbrodt, Cloetta \& Dann, 1978; Richter et al., 2013; Veenman, 1984). Auch die international hohen Abbruchquoten in den ersten Berufsjahren werden auf Anpassungsschwierigkeiten in Form von Stressreaktionen und Burnout-Symptomen der jungen Lehrkräfte zurückgeführt (Schaefer, Long \& Clandinin, 2012; Tynjälä \& Heikkinen, 2011). Das Erleben und Verhalten beim Übergang von der theoretischen zur praktischen Phase werden dabei nach wie vor mit Begriffen wie Praxisschock oder Survival Phase umschrieben (z. B. Stokking, Leenders, de Jong \& van Tartwijk, 2003).

Die empirische Befundlage zu erhöhtem Beanspruchungserleben beim Einstieg in den Lehrerberuf ist dabei weniger eindeutig als es die Diskussion um den Praxisschock impliziert. Tatsächlich gibt es bis dato nur sehr wenige Arbeiten, die dieser Thematik im Kontext des Vorbereitungsdienstes, der in Deutschland den Übergang von der Hochschule in den Beruf einleitet, empirisch nachgehen. Diese ersten Arbeiten weisen auf Anstiege in emotionaler Erschöpfung und körperlichen Beschwerden sowie auf abnehmendes psychisches Wohlbefinden hin (Christ, 2004; Dicke et al., 2015; Klusmann, Kunter, Voss \& Baumert, 2012). In Anbetracht von Reformen des Vorbereitungs-

Psychologie in Erziehung und Unterricht, 2016, 63, 241-243 DOI 10.2378/peu2016.art19d

(c) Ernst Reinhardt Verlag München Basel dienstes in einigen Bundesländern in den letzten Jahren erscheint es ebenfalls notwendig, die Frage nach dem Praxisschock mit aktuellen Daten systematisch zu untersuchen. Überdies mangelt es an Forschung zu geeigneten Strategien, die angehende Lehrkräfte einsetzen könnten, um neuen Herausforderungen des Unterrichtsalltags effektiv begegnen zu können.

Vor diesem Hintergrund war es das Anliegen dieses Themenheftes, aktuelle Arbeiten zu bündeln, die zu einem Verständnis des Erlebens von Stress und Burnout beim Übergang in den Lehrerberuf beitragen. Die Beiträge des Themenheftes zeichnen sich durch ganz unterschiedliche methodische Zugänge aus (längsschnittliche, quasi-experimentelle, Tagebuchund Interventionsstudien) und berücksichtigen verschiedene zeitliche Ausschnitte der Übergangsphase vom Studium über den Vorbereitungsdienst in den Beruf. Inhaltlich gemeinsam ist allen Arbeiten die Untersuchung der zentralen Burnout-Dimension emotionale Erschöpfung, wobei die Beiträge verschiedentlich Erweiterungen vornehmen wie die Berücksichtigung weiterer Burnout-Dimensionen oder positiver Beanspruchungsindikatoren wie Freude am Beruf oder Zufriedenheit mit der Freizeit.

Der erste Beitrag von Dicke, Holzberger, Kunina-Habenicht, Linninger, Schulze-Stocker, Seidel, Terhart, Leutner und Kunter untersucht auf Basis von zwei Kohorten im Längsschnitt die Entwicklung emotionaler Erschöpfung beim Übergang vom Vorbereitungsdienst in den Beruf. In einem quasi-experimentellen Design wird der Frage nachgegangen, ob Faktoren auf Systemebene, wie die zwischen den beiden Kohorten eingeführten Reformen des Vorbereitungsdienstes oder die jeweilige Schulform für die 
Veränderung emotionaler Erschöpfung einen Unterschied machen. Dies scheint nicht der Fall zu sein. Über die Kohorten und Schulformen hinweg steigt für alle Lehrkräfte die Erschöpfung nach Beendigung des Vorbereitungsdienstes leicht an. Diesen Befund deuten die Autorinnen und Autoren dahingehend, dass es am Übergang vom Vorbereitungsdienst in den Beruf zu einem zweiten Praxisschock kommt.

Der zweite Beitrag von Zimmermann, Kaiser, Bernholt, Bauer und Rösler fokussiert auf Entwicklungsverläufe in allen drei BurnoutDimensionen sowie die Bedeutung personaler und sozialer Faktoren über den Verlauf des ersten Jahres im Vorbereitungsdienst. Neben Veränderungen in emotionaler Erschöpfung, $\mathrm{Zy}$ nismus und subjektiv empfundenem Leistungsmangel nach Eintritt in den Vorbereitungsdienst wird auch die Rolle von bereits im Studium bestandenen Ausprägungen in diesen Dimensionen betrachtet. Durchschnittliche Anstiege auf eher niedrigem Niveau zeigen sich für Zynismus und Erschöpfung. Das Erleben von Erschöpfung, Zynismus und Leistungsmangel im Vorbereitungsdienst lässt sich substanziell durch vorher bestandene Ausprägungen in den Burnout-Dimensionen im Studium erklären. Über das Jahr im Vorbereitungsdienst werden konsistent günstige Zusammenhänge der Burnout-Dimensionen mit Lehrer-Selbstwirksamkeitserwartungen, der Zufriedenheit mit den Ausbildungslehrkräften und der sozialen Unterstützung durch angehende Mitlehrkräfte gefunden. Der Befund einer möglicherweise dem Anstieg in Erschöpfung entgegenwirkenden Rolle längerer ausschließlicher Hospitation wird diskutiert.

Der dritte Beitrag von Schmidt, Klusmann und Kunter untersucht zusätzlich zur emotionalen Erschöpfung auch die Freude am Beruf sowie positive und negative Ereignisse in den typischen Tätigkeiten des Lehrerberufs. Es steht die Frage im Mittelpunkt, inwieweit sich in diesen Beanspruchungsindikatoren Hinweise für Anpassungsprozesse beim Berufseinstieg zeigen lassen. Dafür werden auf Basis von Tagebuchdaten drei Kohorten verglichen: angehende Lehrkräfte im ersten und zweiten Jahr des Vorbereitungsdienstes und Lehrkräfte in den ersten fünf Berufsjahren. Ein Kernergebnis ist, dass die Anzahl der positiven berichteten Ereignisse in allen drei Kohorten die Anzahl der negativen Ereignisse im Mittel überwiegt. Die Ergebnisse implizieren auch, dass das Verhältnis von positiven zu negativen Ereignissen insbesondere im Bereich des Unterrichtens über die Zeit günstiger ausfällt. Während sich in diesem Indikator für das Stresserleben eine Verbesserung andeutet, zeigen sich für Freude und Erschöpfung (im Gegensatz zu bisherigen Studien) keine Veränderungen.

Der vierte Beitrag von Bodensteiner beleuchtet in einer prospektiven Interventionsstudie, wie die unter Umständen belastende Unterrichtsvorbereitung effizient bewältigt werden kann, sodass es zu positiven Auswirkungen auf die Zufriedenheit mit der Freizeit kommt. Angehenden Lehrkräften im Vorbereitungsdienst wurden Selbstregulationsstrategien vermittelt, die bei der Unterrichtsvorbereitung eine hilfreiche Ressource darstellen können. Es konnte gezeigt werden, dass der Einsatz von Selbstregulationsstrategien zu geringeren Ausprägungen emotionaler Erschöpfung und im weiteren Verlauf des Vorbereitungsdienstes wiederum zu höherer Zufriedenheit mit der Freizeit beitrug. Der Effekt des Strategieeinsatzes auf die Zufriedenheit mit der Freizeit war dabei vollständig durch die geringere emotionale Erschöpfung vermittelt. Die durch den Einsatz von Selbstregulationsstrategien aufgezeigten positiven Effekte erweisen sich dabei als schulformunabhängig.

Den Abschluss des Themenheftes bildet ein Beitrag von Keller-Schneider, in dem die Autorin über die einzelnen Arbeiten hinausgehend diskutiert, inwieweit Herausforderungen und Beanspruchung am Berufseinstieg adaptive Prozesse auslösen und auch zu Ressourcengewinnen beitragen können.

Insgesamt unterstreichen die empirischen Befunde des Themenheftes die Annahme, dass der Übergang in den praktischen Teil der Ausbildung bzw. in den Beruf eine Anpassungsleistung erfordert, die mit einem erhöhten Stress- 
erleben einhergeht. Allerdings weisen die geringen Effektstärken in den Beiträgen von Dicke et al. und Zimmermann et al. auf einen schwachen mittleren Anstieg im Stresserleben beim Übergang in die Praxis hin. Auch das Überwiegen von positiven im Vergleich zu negativen Ereignissen in den beruflichen Tätigkeiten in der Arbeit von Schmidt et al. deutet an, dass eine Fokussierung auf die negativen Erlebnisqualitäten den psychologischen Prozessen beim Übergang in die Praxis nicht entspricht. Wie gut die Anpassung gelingt, ist dabei auch abhängig von individuellen Merkmalen wie der vorhergehenden Belastung und von selbstregulativen Fähigkeiten, wie sie etwa in der Arbeit von Bodensteiner untersucht werden. Die Beiträge des Themenheftes zeigen, dass der Übergang in die praktische Tätigkeit eine wichtige Phase in der Professionalisierung der Lehrkräfte darstellt. Allerdings sollte kritisch diskutiert werden, ob die Bezeichnung Praxisschock das Erleben und Verhalten der Mehrheit der angehenden Lehrkräfte passend abbildet.

\section{Literatur}

Christ, O. (2004). Die Überprüfung der transaktionalen Stresstheorie im Lehramtsreferendariat. Dissertation, Universität Marburg. Zugriff am 10.11.2015 unter http://archiv.ub.uni-marburg.de/diss/z2005/0197/

Dicke, T., Parker, P. D., Holzberger, D., Kunina-Habenicht, O., Kunter, M. \& Leutner, D. (2015). Beginning teachers' efficacy and emotional exhaustion: Latent changes, reciprocity, and the influence of professional knowledge. Contemporary Educational Psychology, 41, 62-72. http://dx.doi.org/10.1016/j.cedpsych.2014 11.003
Huberman, M. (1989). The professional life cycle of teachers. Teachers College Record, 91, 31-57.

Klusmann, U., Kunter, M., Voss, T. \& Baumert, J. (2012). Berufliche Beanspruchung angehender Lehrkräfte: Die Effekte von Persönlichkeit, pädagogischer Vorerfahrung und professioneller Kompetenz. Zeitschrift für Pädagogische Psychologie, 26, 275-290. http://dx.doi. org/10.1024/1010-0652/a000078

Müller-Fohrbrodt, G., Cloetta, B. \& Dann, H.-D. (1978). Der Praxisschock bei jungen Lehrern. Stuttgart: Klett.

Richter, D., Kunter, M., Lüdtke, O., Klusmann, U., Anders, Y. \& Baumert, J. (2013). How different mentoring approaches affect beginning teachers' development in the first years of practice. Teaching and Teacher Education, 36, 166-177. http://dx.doi.org/10.1016/ j.tate.2013.07.012

Schaefer, L., Long, J. S. \& Clandinin, D. J. (2012). Questioning the research on early career teacher attrition and retention. Alberta Journal of Educational Research, 58(1), 106-121.

Stokking, K., Leenders, F., de Jong, J. \& van Tartwijk, J. (2003). From student to teacher: Reducing practice shock and early dropout in the teaching profession. European Journal of Teacher Education, 26, 329-350. http://dx.doi.org/10.1080/0261976032000128175

Tynjälä, P. \& Heikkinen, H.L.T. (2011). Beginning teachers' transition from pre-service education to working life. Zeitschrift für Erziehungswissenschaft, 14, 11-33.

Veenman, S. (1984). Perceived problems of beginning teachers. Review of Educational Research, 54, 143-178. http://dx.doi.org/10.3102/00346543054002143

\section{Prof. Dr. Friederike Zimmermann}

Institut für Psychologie

Christian-Albrechts-Universität zu Kiel

Olshausenstraße 75

D-24118 Kiel

E-Mail: zimmermann@psychologie.uni-kiel.de

\section{Prof. Dr. Uta Klusmann}

Leibniz-Institut für die Pädagogik

der Naturwissenschaften und Mathematik

Olshausenstraße 62

D-24118 Kiel

E-Mail: klusmann@ipn.uni-kiel.de 